\title{
Enhanced Photoluminescence of Halide Perovskite
}

\author{
Nanocrystals Mediated by a Higher-Order
}

\section{Topological Metasurface}

Alexander S. Berestennikov, ${ }^{+,+}$Anton Vakulenko, ${ }^{\ddagger}$ Svetlana Kiriushechkina, ${ }^{+}$Mengyao Li, ${ }^{+}$

Yanxiu Li,${ }^{\S}$ Lev E. Zelenkov, ${ }^{\ddagger}$ Anatoly P. Pushkarev, ${ }^{*}$ Maxim A. Gorlach, ${ }^{\ddagger}$ Andrey L. Rogach, ${ }^{\S}$ Sergey V. Makarov, ", and Alexander B. Khanikaev"

${ }^{\dagger}$ City College of New York, New York, NY, USA

ITMO University, Saint Petersburg, Russia

$\S$ City University of Hong Kong, Hong Kong SAR, China 


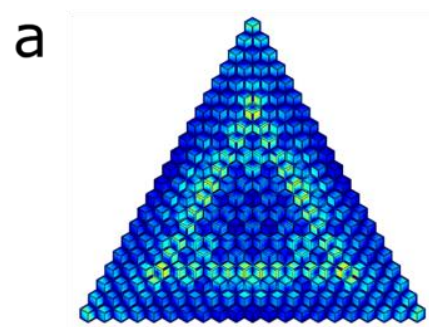

$E=1.9 \mathrm{eV}$ b

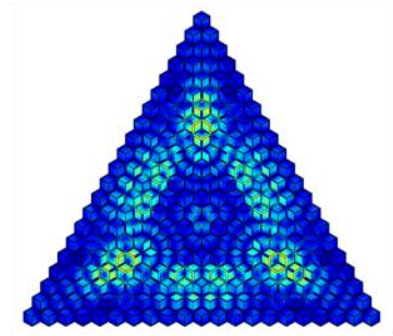

$E=1.93 \mathrm{eV}$
C

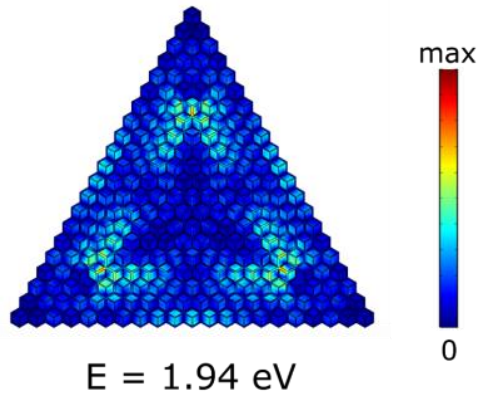

Figure S1. Calculated near-field distributions for topological states of a metasurface without perovskite layer at their corresponding frequencies: edge state (a) and corner states $(b, c)$.
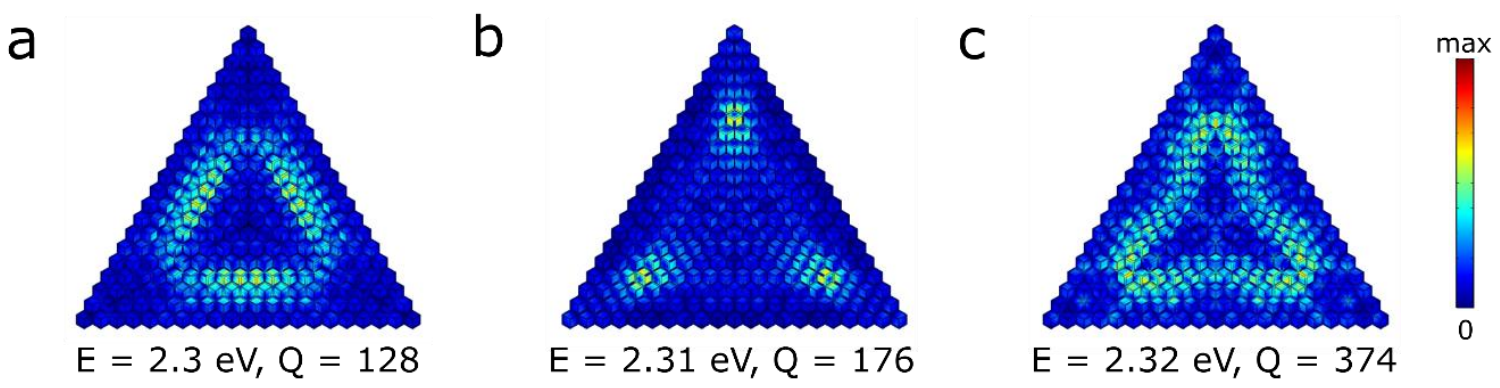

Figure S2. Calculated near-field distributions for topological states of a metasurface based on GaP with perovskite layer at their corresponding frequencies: edge state (a) and corner states $(b, c)$. The period of the structure $a$ is $200 \mathrm{~nm}$, the diamond side length $l_{1}$ is $90 \mathrm{~nm}$, the hexagon side length $l_{2}$ is $115 \mathrm{~nm}$, and total height of structure $h$ is $90 \mathrm{~nm}$. Unit cell of internal kagome lattice was expanded by $5 \%$, and the external lattice was shrunken by $10 \%$ relatively to the unperturbed lattice. 


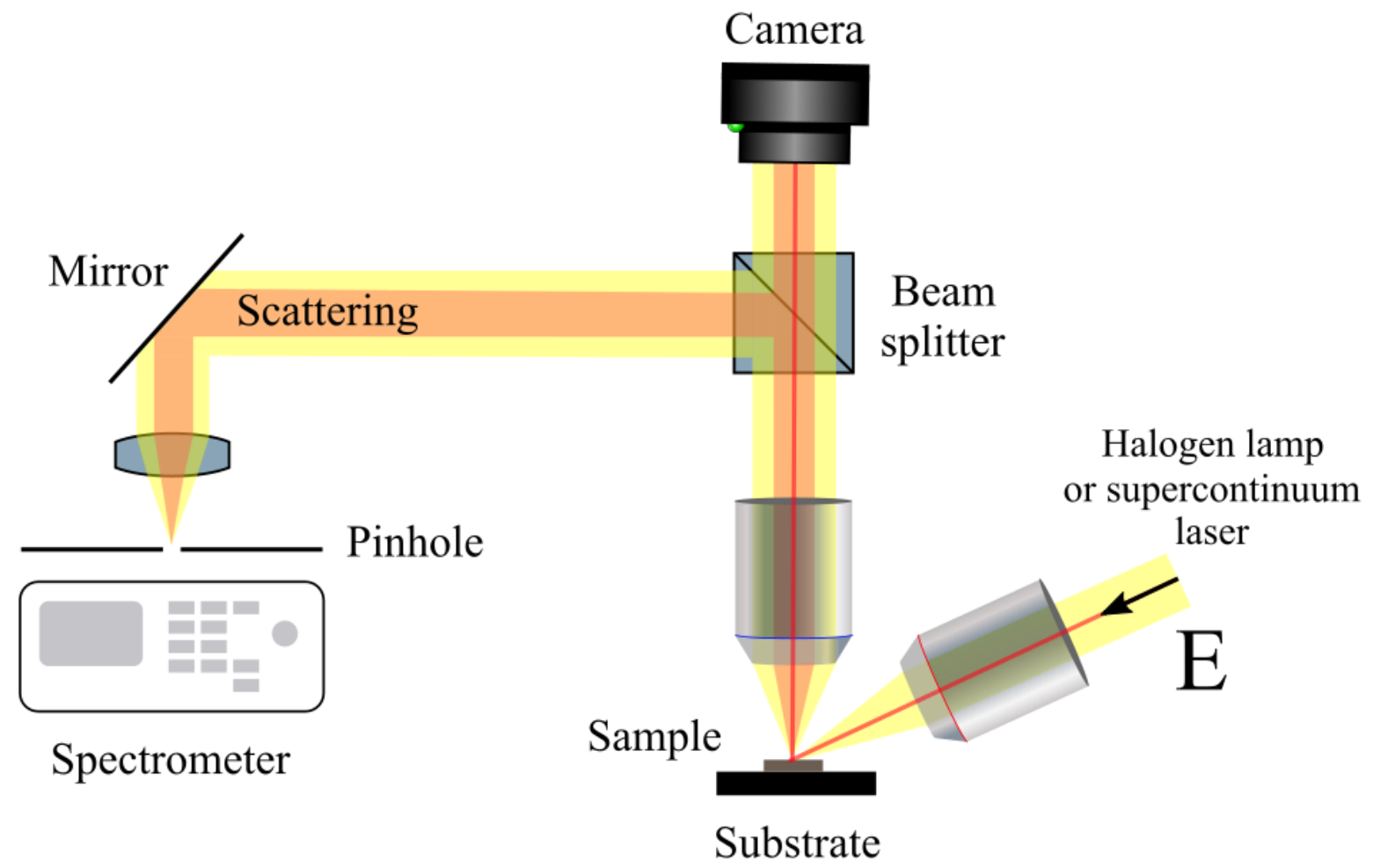

Figure S3. Optical scheme of setup for dark-field measurements. 


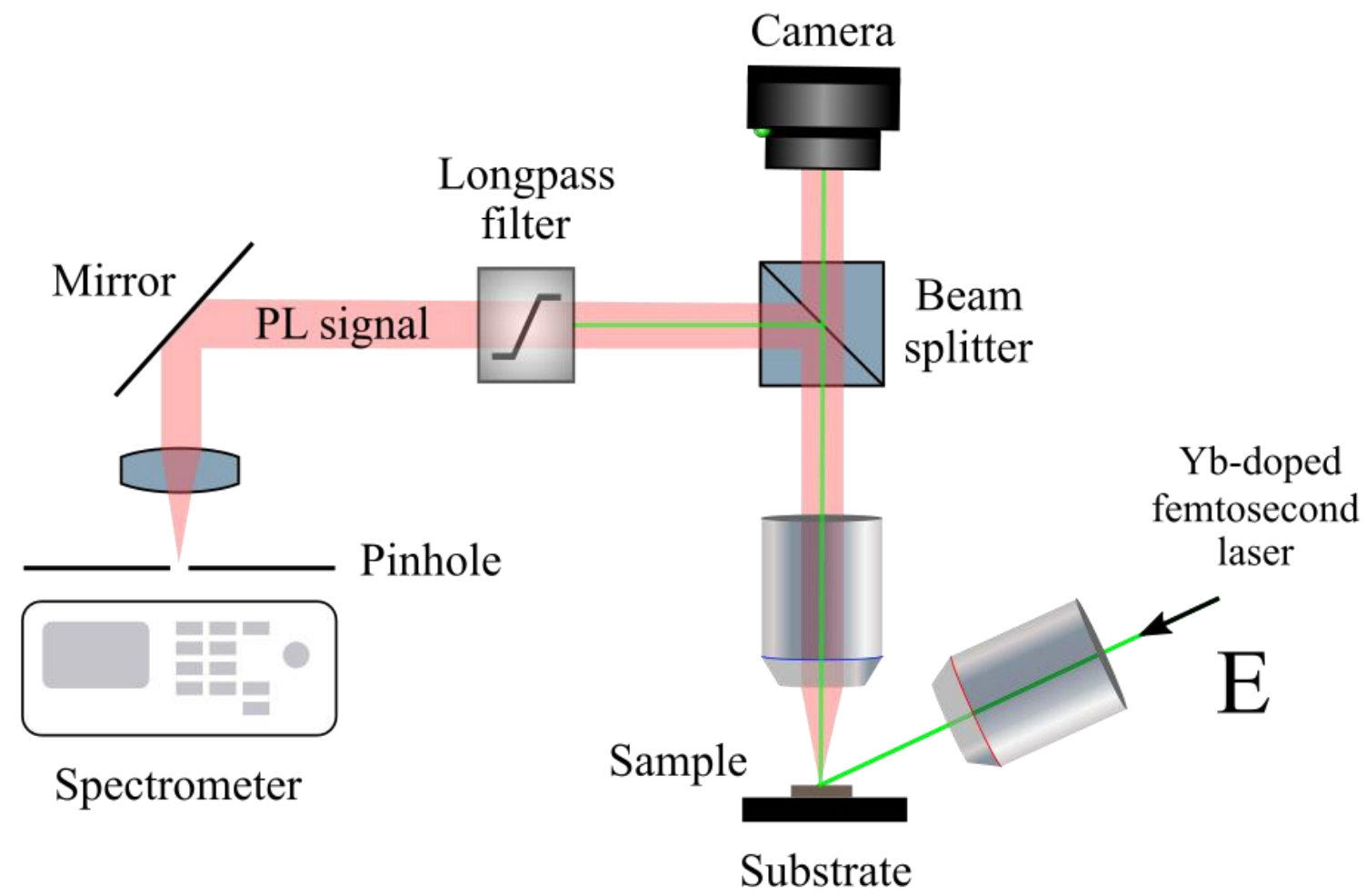

Figure S4. Optical scheme of setup for photoluminescence measurements. 